\section{The magnitude of sensory preconditioning as a function of the time interval between stimulus onsets}

\author{
CHESTER L. OLSON* \\ Waterloo Lutheran University, Waterloo, Ontario, Canada
}

A light and a tone were presented unpaired or paired with onset intervals of $-.5,0, .5$, or 1 sec to independent groups of 18 male students. For all Ss, the second stage involved classical conditioning of GSR to tone, and the third stage tested for sensory preconditioning (SPC) in extinction trials to light. The results indicated that no SPC occurred, but on two test trials there was a significant quadratic component in the relationship of difference scores to the preconditioning interstimulus interval. It is suggested that Ss' hypotheses about stimulus relations should be considered in SPC researeh with humans.

Sensory preconditioning (SPC) is defined by three stages of operations: (1) the preconditioning (PC) stage, consisting of paired presentations of two presumably neutral stimuli; (2) the conditioning stage, in which a CR is established to one of the stimuli; and (3) the test stage, in which the transfer of the CR to the other stimulus defines SPC.

Most of the early SPC research tended to use simultaneous stimulus onsets in the PC stage, but disputes between $S-R$ and $S-S$ contiguity learning theorists made the question of the effectiveness of different PC interstimulus intervals (ISIs) theoretically relevant. The results of previous research have been inconsistent in this respect, and both positive and negative results have been published for the three basic types of pairings of stimuli: backward, simultaneous, and forward. Studies with cats as Ss (e.g., Wynne \& Brogden, 1962) have established SPC in the range from simultaneous stimulus onset to an ISI of $4 \mathrm{sec}$, with the maximum effect at 4 sec. Wickens \& Cross (1963) used GSR as the response measure with human Ss and found that the amount of SPC increased as the ISI increased from $0 \mathrm{msec}$ to $400 \mathrm{msec}$, where the effect was maximal, beyond which SPC magnitude was less at $600 \mathrm{msec}$ than at $0 \mathrm{msec}$. The fact that they used no control group, however, leaves some question as to which groups actually showed any SPC at all.

The present study investigated various PC ISIs in human Ss, with GSR as the response measure. Specifically, the use of ISIs of $-500,0,500$, and 1,000 msec defined four experimental groups, while a control group received unpaired PC stimulation. It was hypothesized (1) that there would be

* Now at the University of Toronto, Toronto, Ontario, Canada. greater test stage responses in Ss with a 500-msec ISI than in Ss with an ISI of 1,000 msec, whose test stage responses would in turn be greater than those of Ss with ISIs of -500 or $0 \mathrm{msec}$ or with unpaired stimulation, and (2) that there would be a quadratic component in test stage response magnitude plotted as a function of PC ISI, such that the best-fitting curve would be a downward-opening quadratic with a maximum at approximately $500 \mathrm{msec}$. SUBJECTS

Ss were 90 male volunteers from an introductory psychology course. A randomized block design was used to form five groups of 18 Ss each.

\section{APPARATUS}

A tone and a light were used as stimuli in the PC stage. The tone was $1,000 \mathrm{~Hz}$, fed into earphones so as to yield a sound-pressure level of $40 \mathrm{~dB}$ (re .0002 microbar). The light was a 7.5- $\mathrm{W}$ white frosted bulb, located in a vertical flat-black panel about $2 \mathrm{ft}$ directly in front of $S$. A Grason-Stadler shock generator (Model 700) was used to deliver shocks between .05 and $3 \mathrm{~mA}$ at $60 \mathrm{~Hz}$. The durations of tone, light, shock, and ISI were controlled by four decade interval timers.

Two electrodes were fastened to the right hand to deliver shock. Electrodes were also attached on the left-hand side to the dorsum of the forearm and to the volar distal phalanx of the middle finger to register variations in voltage drop across $\mathrm{S}$ due to changes in skin resistance. The laboratory was dimly illuminated by indirect lighting, minimized external noise.

\section{PROCEDURE}

Each $S$ reported to the laboratory left arm strapped to the arm of the chair. As the electrodes were being fitted, $\mathbf{S}$ read the initial instructions, which outlined the purpose of the volar surface of the little finger of S's and $S$ wore padded earphones which individually and was seated with his electrodes and explained that the shock intensities used in the experiment itself would depend on the lowest current level $S$ was able to perceive as $\mathrm{E}$ gradually increased the amperage in a preexperimental trial. After determination of shock intensity, the final instructions told S that his task was simply to remain alert to notice the stimulus light, tone, and electric shock sensation when each occurred. Questions other than those concerning the sequence of stimulus presentations were answered factually, and the first $\mathrm{PC}$ event began after a 2-min interval.

For each group there were three stages, PC, classical conditioning (CC), and test, with an interval of $1 \mathrm{~min}$ between stages. The PC stage involved 10 light and 10 tone presentations for each group. For Groups 0,500, and 1,000 , the light onset was 0,500 , and $1,000 \mathrm{msec}$, respectively, before the onset of a 500 -msec tone; the stimuli had simultaneous offsets. Group -500 was the same as Group 500, except that, in the former, the tone began $500 \mathrm{msec}$ before the light rather than vice versa. In Group C a 500-msec light and a 500-msec tone were presented in a different prearranged random order for each $\mathrm{S}$, with neither tone nor light appearing more than three times consecutively. For Group C the randomly ordered intertrial intervals (ITIs) in the PC stage were 10,15 , and $20 \mathrm{sec}$, with a mean of $15 \mathrm{sec}$. For all other groups the mean duration of the ITIs in the PC stage was 30 sec.

The CC stage consisted of 10 presentations of a $600-\mathrm{msec}$ tone, concurrent for the last $100 \mathrm{msec}$ with a shock; the mean ITI was $30 \mathrm{sec}$. To compensate for habituation, the shock intensity was increased over the 10 trials, with the individually determined current levels typically being between 1 and $2 \mathrm{~mA}$.

The test stage consisted of five presentations of a $500-\mathrm{msec}$ light, with a mean ITI of $30 \mathrm{sec}$.

The resistance level in kilohms was recorded at the onset of each stimulus or stimulus pair and again at its lowest value in the next $10 \mathrm{sec}$. The values were converted to a logarithmic conductance scale (log micromhos), and the difference between the log conductance scores so obtained was considered to be the GSR to that stimulus or stimulus pair.

\section{RESULTS}

Figure 1 shows the mean responses in each of the three stages of the experiment plotted as a function of the duration of the PC ISI. The mean responses of Group C in each stage provide a comparison for the other response levels.

The mean responses in the PC stage were analyzed across the four 


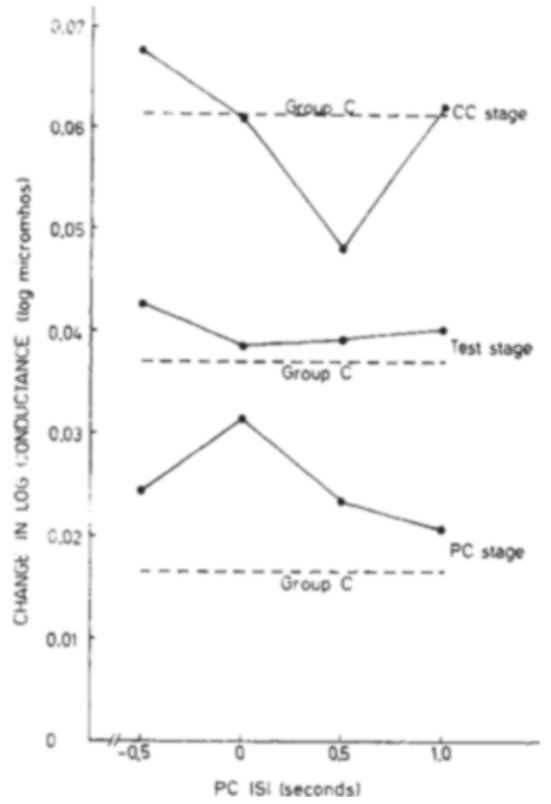

Fig. 1. Mean GSRs in each experimental stage as a function of $\mathrm{PC}$ ISI duration.

experimental groups to indicate whether or not the groups differed in GSR reactivity at this point in the experiment. Group $\mathrm{C}$ was not included in this analysis, since Group C Ss had received 20 simple stimulus presentations in the $\mathrm{PC}$ stage, whereas Ss in the other groups had received 10 compound stimulus presentations. The analysis of variance showed no significant differences in GSR reactivity between groups.

Similarly, an analysis of variance conducted with the mean responses in the CC stage provided no evidence of significant differences in GSRs during the second stage of the experiment.

The absence of significant differences in responses in the $\mathrm{CC}$ stage allowed the use of mean GSRs in $\mathrm{CC}$ as a covariate in the analysis of results in the test stage. The only significant finding in the two-way analysis of covariance with repeated measures on one variable was the main effect due to test trials $[F(4,339)=22.93, \quad p<.01] . \quad A$ Newman-Keuls analysis demonstrated that the mean response on the first test trial was significantly greater than that on each of the succeeding test trials $(p<.01)$, none of which differed from each other.

Because of the significant main effect due to test trials, separate analyses of covariance were performed on the data from each test trial. There was no significant effect due to PC ISI groups on any of the test trials.

One purpose of the present study was to investigate the shape of the curve relating magnitude of response in the test stage to the PC ISI.
However, the graph of test stage response magnitude as a function of PC ISI is distorted by the variability of GSRs in the CC stage. Consequently, for the orthogonal polynomial analyses of the relationship between response magnitude in the test stage and PC ISI duration, the response unit was considered to be the decrease in response magnitude from the $\mathrm{CC}$ stage to the particular test trial. Difference scores were calculated for each $\mathrm{S}$ on each test trial by subtracting his test trial response magnitude from his mean GSR in the CC stage. An orthogonal polynomial analysis was conducted on the difference scores for each test trial. The only significant findings were quadratic components on the third and fifth test trials $(p<.05)$. The best-fitting curves, which opened upward because of the use of difference scores, showed greatest responding at PC ISIs of 340 and $350 \mathrm{msec}$ on Trials 3 and 5 , respectively.

\section{DISCUSSION}

Because the nonsignificant analyses of covariance included comparisons of Group $C$ with the experimental groups, it is clear that there was no evidence of SPC in any group nor any experimental support for the first hypothesis of the present study.

The one significant finding in the repeated-measure analysis of covariance, the within-S effect due to test trials, reflected the higher responding on Trial 1 as compared with each of the other four trials. Since no SPC was established in this study, this result cannot be attributed to the extinction of SPC in the test trials, but it is easily accounted for in terms of the phenomenon known as the perceptual disparity response (Grings, 1960). Allen, Hill, \& Wickens (1963) presented strong evidence that any change in procedure, such as from training to extinction trials, can produce an increase in GSR as a perceptual disparity response.

The second hypothesis of the present study was not supported, insofar as it predicted a quadratic relationship between the actual test stage responses and PC ISIs. However, some partial support is seen in the difference score analyses on the third and fifth test trials. Except for the inversion of the curves due to the use of difference scores in the present study, the quadratic relationships found here, which predict maximum responding at about $350 \mathrm{msec}$, are compatible with the finding by Wickens \& Cross (1963) that maximum responding occurred with a PC ISI of 400 msec. Wickens and Cross, who did not use a control group, reported their results as relating SPC to PC ISI. It is interesting to note that a similar relationship was obtained in the present stidy, in which the evidence suggests that no SPC was established in any group.

The most plausible interpretation of the failure to establish SPC in the present study was suggested by the spontaneous comments of Ss following the experimental sessions. These comments indicated expectations varying over $S$ s concerning the nature of the experiment. It is relevant to this interpretation that the hypothesized relationship between test stage response magnitude and PC ISI was present to some degree, as evidenced by the findings of the orthogonal polynomial analyses, but with human Ss a much more potent variable appears to be $S$ 's conceptualization of the sequence of stimulus presentations. The conclusion of Zeiner \& Grings (1968) concerning backward conditioning in humans would seem to be applicable to work in SPC as well. They contended that conditioned responses in humans are not straightforward associations between stimuli but are complicated by S's conceptual framework. As applied to SPC, this implies that test stage response magnitude is largely a function of S's expectations, which in turn are based chiefly on his ideas about the first two stages. Such expectations are presumably relatively independent of PC ISI, and, in general, would tend to outweigh the effect of other variables influencing SPC magnitude.

\section{REFERENCES}

ALLEN, C. K., HILL, F. A., \& WICKENS D. D. The orienting reflex as a function of the interstimulus interval of compound stimuli. Journal of Experimental Psychology, 1963, 65, 309-316.

GRINGS, W. W. Preparatory set variables related to classical conditioning of autonomic responses. Psychological Review, 1960, 67, 243-252.

WICKENS, D. D., \& CROSS, H. A. Resistance to extinction as a function of temporal relations during sensory preconditioning. Journal of Experimental Psychology, 1963, 65, 206-211.

WYNNE, J. D., \& BROGDEN, W. J. Supplementary report: Effect upon sensory preconditioning of backward, forward, and trace preconditioning training. Journal of Experimental Psychology, 1962, 64, 422-423.

ZEINER, A., \& GRINGS, W. W. Back ward conditioning: A replication with emphasis on conceptualizations by the subject. Journal of Experimental Psychology, $1968,76,232-235$. 